\title{
Pinta: A System for Visualizing the Anatomical Structures of the Brain from MR Imaging
}

\author{
B. Parvin, W. Johnston and D. Roselli \\ Information and Computing Sciences Division \\ Lawrence Berkeley Laboratory \\ University of California \\ Berkeley, CA 94720
}




\section{DISCLAIMER}

This report was prepared as an account of work sponsored by an agency of the United States Government. Neither the United States Government nor any agency thereof, nor any of their employees, make any warranty, express or implied, or assumes any legal liability or responsibility for the accuracy, completeness, or usefulness of any information, apparatus, product, or process disclosed, or represents that its use would not infringe privately owned rights. Reference herein to any specific commercial product, process, or service by trade name, trademark, manufacturer, or otherwise does not necessarily constitute or imply its endorsement, recommendation, or favoring by the United States Government or any agency thereof. The views and opinions of authors expressed herein do not necessarily state or reflect those of the United States Government or any agency thereof. 


\section{DISCLAIMER}

Portions of this document may be illegible in electronic image products. Images are produced from the best available original document. 


\title{
Pinta: A System for Visualizing the Anatomical Structures of the Brain from MR Imaging
}

\author{
B. Parvin, W. Johnston and D. Roselli \\ Information and Computing Sciences Division \\ Lawrence Berkeley Laboratory \\ University of California \\ Berkeley, CA 94720
}

\begin{abstract}
Pinta is a system for segmentation and visualization of anatomical structures obtained from serial sections reconstructed from Magnetic Resonance Imaging. The system approaches the segmentation problem by assigning each volumetric region to an anatomical structure. This is accomplished by satisfying constraints at the pixel level, slice level, and volumetric level. Each slice is represented by an attributed graph, where nodes correspond to regions and links correspond to the relations between regions. These regions are obtained by grouping pixels based on similarity and proximity. The slice level attributed graphs are then coerced to form a volumetric attributed graph, where volumetric consistency can be verified. The main novelty of our approach is in the use of the volumetric graph to ensure consistency from symbolic representations obtained from individual slices. In this fashion, the system allows errors to be made at the slice level, yet removes them when the volumetric consistency cannot be verified. Once the segmentation is complete, the $3 \mathrm{D}$ surfaces of the brain can be constructed and visualized.
\end{abstract}

\section{Introduction}

Pinta is a system designed for automatic segmentation, visualization, and description of the human brain from Magnetic Resonance Images. The vitimate objective of this system is to provide a research vehicle for gaining new insights into the structural changes over time and across individuals. In this paper, we focus on the segmentation and visualization aspects of our system, since the volumetric description is the subject of our continued research. In practice, MR images can be tuned to accentuate certain anatomical structures. For example, by applying a specific pulse sequence, the boundary separation between cerebrospinal fluid (CSF) gray and white matters can be maximized. From this perspective, the segmentation procedure should label each pixel in the data volume accordingly. Our approach to the segmentation process is to exploit the knowledge of the anatomical structures coupled with the image characteristics and arrive at a correct labeling. In this sense, the segmentation is model driven, where the model is expressed in terms of anatomical constraints and certain knowledge about the image formation. From an anatomic standpoint, the cerebral cortex is a single sheet of convoluted structure. And from the image formation perspective, certain intensity distributions reflect the presence of CSF and white and gray matter. Hence, we have organized various constraints at different levels of

- Research was supported by the U.S. Dept. of Energy, Office of Scientific Computing, under Contract DE-AC03-76SF00098 the computational hierarchy so that stable labeling can be realized. These constraints are imposed on homogeneous patches that are extracted by clustering and relaxation. The clustering step estimates the center of the mass for each class, and the relaxation step ensures local consistency. The system applies the necessary verification step to ensure that the result of clustering is anatomically correct (based on the knowledge of image intensity distribution in the cortex). Each slice of the image is represented with an attributed graph, where nodes correspond to regions and links correspond to the relations between regions. These attributed graphs are then coerced together to form a volumetric attributed graph, where $3 D$ consistency can be verified. In this manner, the system tolerates erroneous labeling at the slice level, and then removes them at higher level of processing. Due to space limitation, we will not survey the previous work, however, a brief summary can be found in [2]. We now outline the details of our approach followed by results on real data.

\section{Description of the method}

There are two major components to our system. The first one operates on slice level information and generates an attributed graph. In this component, local consistency at the pixel level, together with the region consistency, is enforced to reduce the number of potential hypotheses. The second component operates on the volumetric attributed graph and ensures $3 D$ consistency. This is shown in figure 1 , where the feedback loop ensures correct segmentation at each level of the hierarchy. The segmentation technique is model driven, where the model is represented in terms of anatomical constraints. For example, we know that ventricles are present at a certain distance from the top of the skull. These modeling cues are used to initiate the segmentation process from a particular slice called the seed frame. In the seed frame, the ventricles are detected through their intensity distribution and shape symmetries and then tracked in consecutive slices.

\subsection{Intra-slice subsystem}

There are two parts in this subsystem. The first one groups neighboring pixels with similar properties (low level processing) and builds an initial symbolic description. The second part searches for a particular structure and corrects for erroneous labeling. A careful analysis of MRI data, obtained in the T1-relaxation mode, reveals that four-class region segmentation is sufficient for extracting the important patches from brain scans. These correspond to white matter, gray matter, CSF and others. The architecture for the intra-slice subsystem is shown in figure 2 , and examples of this step of computational process are shown in figure 


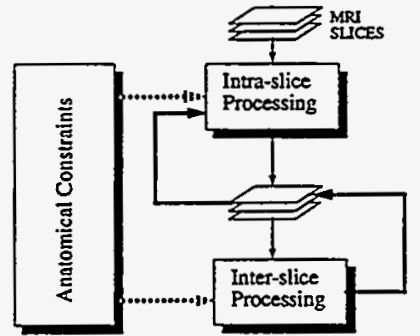

Figure 1: System Architecture

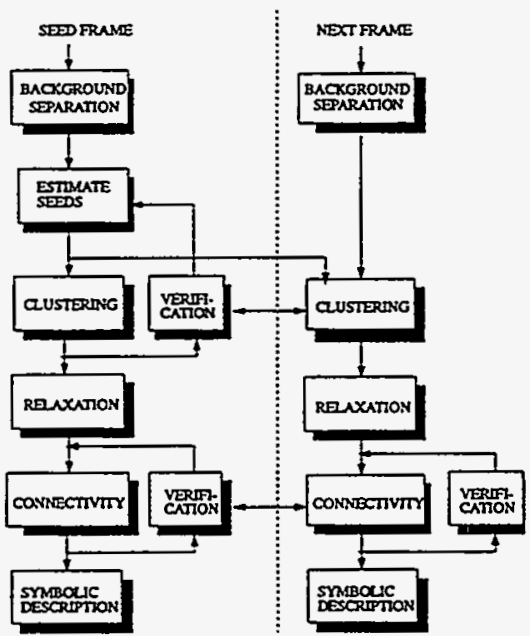

Figure 2: Intra-slice Processing

3. Where contours of different regions (tissues) are overlied on top of the original image. Note that some of the membrane tissues are also labeled as white matter at the slice level labeling; however, these errors will be corrected at the higher level process.

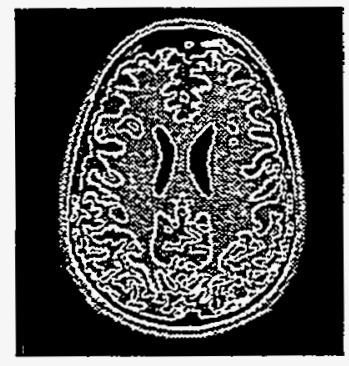

(a)

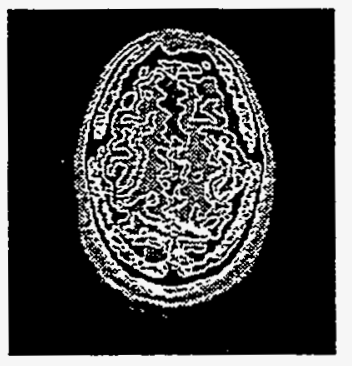

(b)
Figure 3: Slice level grouping for two slices

\subsection{Inter-slice subsystem}

At the completion of the previous stage, the content of each slice is symbolically represented as an attributed graph. The inter-slice process goes beyond the evidences in a given slice and attempts to resolve ambiguities that can be corrected through volumetric analysis. For example, we have already indicated that the white matter is a singly convoluted structure. This anatomical constraint translates into $3 \mathrm{D}$ connectivity among all regions that are labeled as white matter in each slice. Thus, any erroneously labeled region can be removed using a simple binary constraint.
An example is shown in figure 4 indicating the correct segmentation of the white matter.

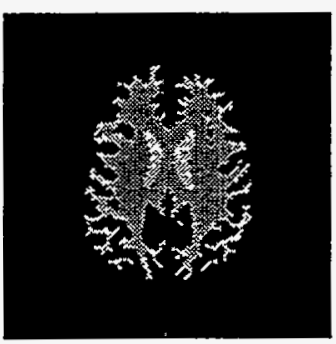

(a)

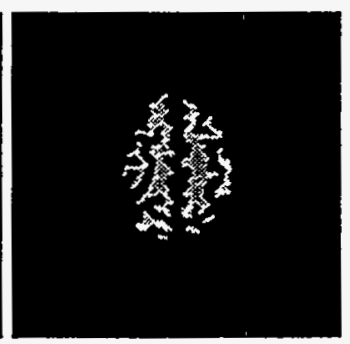

(b)
Figure 4: Volumetric level grouping for two slices 2.3 Visualization from serial sections

Visualization has been the subject of many researchers. The simplest way to compute the $S D$ surfaces is by triangulation between adjacent pairs of contours. This is adequate for surfaces that are almost convex. Yet, triangulation becomes ambiguous when the surface topology changes rapidly. An alternative is to fit partial surfaces into a small region. An example of this includes Marching Cubes [1] with linear interpolation between adjacent samples. Our system uses the segmented volumetric information to produce a polygonal representation of the surfaces using the Marching Cubes, which can also be used for volumetric rendering. Figure 5 shows the result of surface reconstruction from 60 frames of $M R$ images.

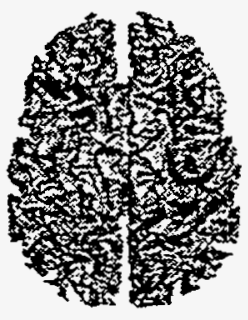

(a)

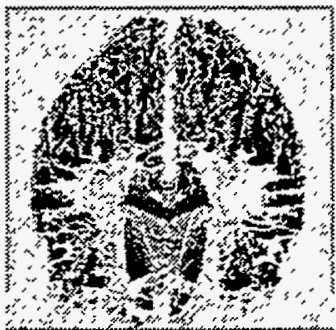

(b)
Figure 5: Surface reconstruction and rendering from two views: (a) white matter; (b) two isosurfaces corresponding to white and ventricle.

\section{Conclusion}

In this paper, we have presented a unique computational scheme for segmentation of Magnetic Resonance Images. The results were then used for rendering and visualization. The system achieves reliable segmentation by enforcing constraints at three levels of hierarchies; these include the pixel level, the slice level, and volumetric level. Our current research focuses on the description of these complex superstructures and how they relate to one another.

Acknowledgments: The authors thank Dr. Tom Budinger and Greg Klein for supplying the data for this research.

\section{References}

[1] W. Lorensen and H. Cline, "Marching Cubes: A High Resolution 3D Surface Reconstruction Algorithms," Computer Graphics, Vol. 21, 1987, pp.163-169.

[2] B. Parvin, W. Johnston and D. Roselli, "Pinta: A System for Visualizing the Anatomical Structures of the Brain from MR Imaging," $L B L$ Internal Report, 1992. 